\title{
A projection-domain iterative algorithm for metal artifact reduction by minimizing the total-variation norm and the negative-pixel energy
}

Gengsheng L. Zeng ${ }^{1,2}$

\begin{abstract}
Metal objects in X-ray computed tomography can cause severe artifacts. The state-of-the-art metal artifact reduction methods are in the sinogram inpainting category and are iterative methods. This paper proposes a projectiondomain algorithm to reduce the metal artifacts. In this algorithm, the unknowns are the metal-affected projections, while the objective function is set up in the image domain. The data fidelity term is not utilized in the objective function. The objective function of the proposed algorithm consists of two terms: the total variation of the metalremoved image and the energy of the negative-valued pixels in the image. After the metal-affected projections are modified, the final image is reconstructed via the filtered backprojection algorithm. The feasibility of the proposed algorithm has been verified by real experimental data.
\end{abstract}

Keywords: Analytical image reconstruction, Metal artifact reduction, Projection-domain iterative algorithm, X-ray computed tomography

\section{Introduction}

The metal artifacts in X-ray computed tomography (CT) are mainly due to the beam hardening effects [1-4]. The linear attenuation coefficient in a material is a function of the X-ray energy. X-ray CT systems normally use polychromatic beams, and the various energy components are not attenuated uniformly. The lower energy component of X-ray spectrum is more easily to be attenuated or even completely adsorbed when traveling through metals. The beam hardening effects make the sinogram values deviate from the assumption that the sinogram values are the line-integrals of the attenuation coefficients in the object.

Many techniques are commonly used to reduce the metal beam hardening effects. In clinical scans, the metal

Correspondence: larry.zeng@uvu.edu

'Department of Computer Science, Utah Valley University, Orem, UT 84058, USA

${ }^{2}$ Department of Radiology and Imaging Sciences, University of Utah, Salt Lake City, UT 84108, USA

\section{Springer Open}

materials and the X-ray source settings are known. Model-based iterative algorithms [5-12] and iterative segmentation-based interpolation methods [13-20] can be used by taking the advantage of the available knowledge of the metals, the soft tissues, the bones, and X-ray source settings.

In this paper, we consider a more challenging situation where the objects of interest, the metal materials and the X-ray source settings are all unknown. Thus, model-based iterative reconstruction methods are not effective here.

There are other methods to be considered. Bayesian algorithms, for example, the total variation (TV) norm minimization can be considered [21-25]. The metal artifacts appear as bright or dark streaks, radiated from the metals. The bright parts are the positive overshoots; the dark parts are the negative undershoots. The negative undershoots may result in negative image pixels. Our proposed algorithm is to minimize the TV of the metalremoved image [25] and the energy of the negative

(c) The Author(s). 2021 Open Access This article is licensed under a Creative Commons Attribution 4.0 International License, which permits use, sharing, adaptation, distribution and reproduction in any medium or format, as long as you give appropriate credit to the original author(s) and the source, provide a link to the Creative Commons licence, and indicate if changes were made. The images or other third party material in this article are included in the article's Creative Commons licence, unless indicated otherwise in a credit line to the material. If material is not included in the article's Creative Commons licence and your intended use is not permitted by statutory regulation or exceeds the permitted use, you will need to obtain permission directly from the copyright holder. To view a copy of this licence, visit http://creativecommons.org/licenses/by/4.0/. 
image pixels [26]. The algorithm variables are the metal affected sinogram values.

We must point out that it is not straightforward to reflect the negative image pixel constraint in the sinogram domain. It is not equivalent to setting the associated sinogram values to zero or positive [26].

\section{Methods}

This paper is inspired by the two observations that the metal artifacts often result in dark/bright streaks radiating from the metals [25] and negative image pixels around the metals [26]. The streaking artifacts increase the TV norm of the non-metal regions of the image. The attenuation coefficients cannot be negative. These two effects were dealt with in two papers, separately [16, $26]$. The goal of this current paper is to combine these two methods into one.

Let the measured sinogram (i.e., line integrals) be $P$, which can be divided into two parts: the metal affected part $P_{M}$ and the metal unaffected part $P_{n o t M}$. This separation can be readily achieved by performing a raw filtered backprojection (FBP) reconstruction, thresholding, and forward projection. The objective function is hence

$$
\begin{aligned}
& \text { Objectivefunction }=T(P)=\beta_{1} T_{1}+\beta_{2} T_{2} \\
& =\beta_{1}\|G\{F(P)\}\|_{T V}+\beta_{2}\|\min \{0, F(P)\}\|^{2}
\end{aligned}
$$

where the first term is the TV norm, and the second term is the squared $L_{2}$ norm. In Formula (1), $F$ denotes the FBP reconstruction operator, $G$ is the operator that removes the metals from the image, and $\beta_{1}$ and $\beta_{2}$ are the parameters balancing the two norms. In the gradient descent algorithm, $\beta_{1}$ and $\beta_{2}$ also act as the step sizes (also, known as the relaxation factors).

We minimize this objective function with respect to unknowns $P_{M}$ with a gradient descent algorithm. It is noticed that neither of the two terms in Formula (1) is differentiable. Sub-gradients are used to combat this difficulty [27].

A sub-gradient is a 'surrogate' of the gradient: it coincides with the gradient whenever a gradient exists, and it generalizes the notion of gradient at points where the function is non-differentiable. Let us consider a point of interest and the function is non-differentiable at this point. A sub-gradient is evaluated as follows. We find the left-gradient by taking the limit from the left and the right-gradient by taking the limit from the right, which are also known as the directional gradients. Any values between these two left and right values can be used as the sub-gradient.

We now use the absolute function $|x|$ to illustrate how to find the sub-gradient. When $x>0$, the subgradient is the same as the gradient: 1 . When $x<0$, the sub-gradient is the same as the gradient: -1 .
When $x=0$, the function $|x|$ is non-differentiable. The left-gradient is -1 and the right-gradient is 1 . The sub-gradient at $x=0$ can be any value in the interval $[-1,1]$.

In our implementation, three values of $\beta_{1}$ were tested: 0 , 0.002 and 0.004; two values of $\beta_{2}$ were tested: 0 and 5 .

\section{The FBP algorithm}

In Formula (1), $F(P)$ is a linear algorithm that can be deposed into two steps. The first step is to convolve the sinogram $P$ along the detector dimension with a convolution kernel $h(n)$ :

$$
h(n)=\left\{\begin{array}{ccc}
\frac{1}{4} & \text { if } & n=0 \\
-\frac{1}{(n \pi)^{2}} & \text { if } & n \text { is odd } \\
0 & \text { otherwise. } &
\end{array}\right.
$$

The second step is the backprojection. Let the FBP reconstruction from $P$ be $X$. If both $P$ and $\mathrm{X}$ are represented in the vector forms, $F(P)$ can be written as matrix multiplication

$$
X=F P
$$

where $F$ is a matrix.

For a given threshold value $t$, a metal image is obtained by modifying the image $X$. If a pixel in $X$ is greater than $t$, its value is set to 1 , otherwise its value is set to 0 . The forward projection of $X$ generates a 'shadow' in the projection domain. If the shadow values are positive, the corresponding sinogram values are called metal-affected projections, $P_{M}$. If the shadow values are zero, the corresponding sinogram values are called metal-not-affected projections, $P_{\text {not } M}$. The sinogram $P$ is thus divided into two parts: $P_{M}$ and $P_{\text {not } M}$. The projection values in $P_{M}$ are treated as variables in the proposed algorithm.

In Formula (1), the metal removed FBP image, $G\{F(P)\}=G\{X\}$, is almost the same as $X$, except that if a pixel value is greater than $t$, its value is set to 0 .

In Formula (1), $\min \{0, F(P)\}=\min \{0, X\}$ is almost the same as $X$, except that if a pixel value is positive, its value is set to 0 . Here, the 'min' function is an elementwise function.

\section{Optimization of the objective function (Formula 1) by the gradient descent algorithm}

A gradient descent algorithm to minimize the objective function $T(P)$ in Formula (1) can be expressed as

$$
P_{M}^{(k+1)}=P_{M}^{(k)}-D\left[\beta_{1} \nabla T_{1}(P)+\beta_{2} \nabla T_{2}(P)\right]
$$

where the super script $(k)$ is the iteration index. The projection vector $P$ consists of two parts: the metal affected 
part $P_{M}$ and the metal not-affected part $P_{\text {not } M}$. The metal not-affected part $P_{\text {notM }}$ does not get updated from iteration to iteration. The matrix $D$ in Formula (4) is a diagonal matrix with 0 's and 1's, which discards the entries in $P_{n o t M}$. The parameter $\beta_{1}$ and $\beta_{2}$ in Formula (4) controls the step sizes of the gradient descent algorithm for the two different norms, respectively. In Formula (4), $\nabla T_{1}$ is the gradient of the TV norm of the image with the metals removed, and $\nabla T_{2}$ is the gradient of the energy of the negative pixels in the image. These two gradients are calculated with respect to the projections $P$. These two gradients are discussed further in the following two sub sections.

\section{Minimization of the first term in the objective function} (Formula 1)

Let $Y=G\{F(P)\}=G\{X\}$ be the metal-removed FBP reconstruction. The image $Y$ is represented in a twodimensional array and $y_{i, j}$ is its pixel value at the $i$ th row and $j$ th column. The TV norm of $Y$ is defined as

$$
T_{1}=\sum_{i, j} \sqrt{\left(y_{i, j}-y_{i, j+1}\right)^{2}+\left(y_{i, j}-y_{i+1, j}\right)^{2}}
$$

The partial derivative of $T_{1}$ with respect to pixel $(i, j)$ (if exists) is readily calculated as

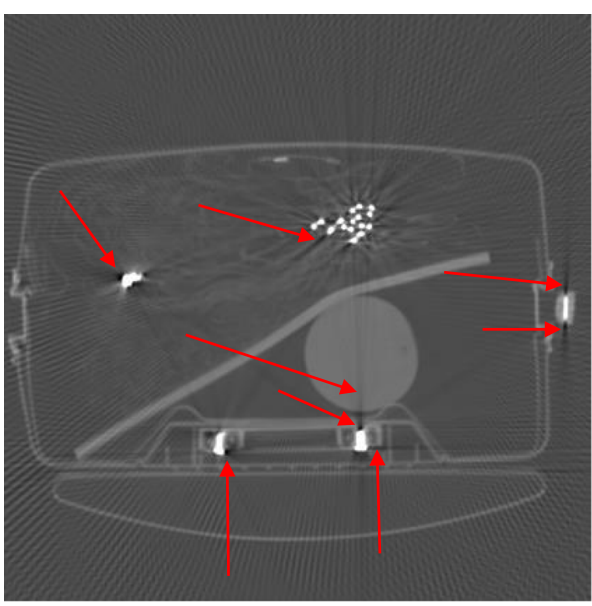

(a)

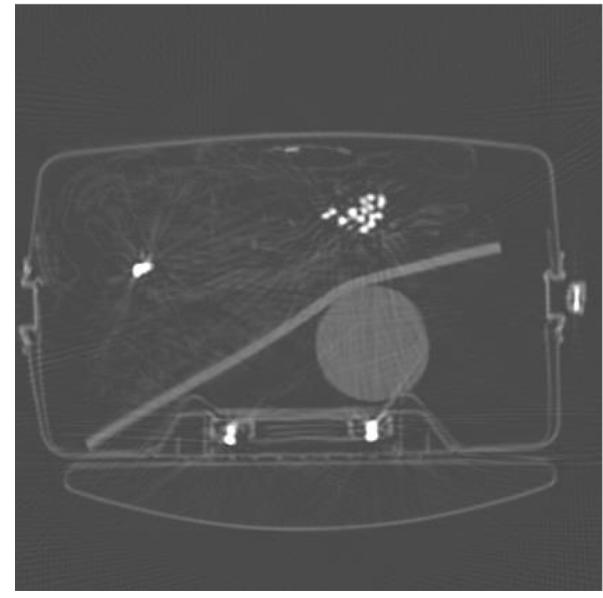

(c)

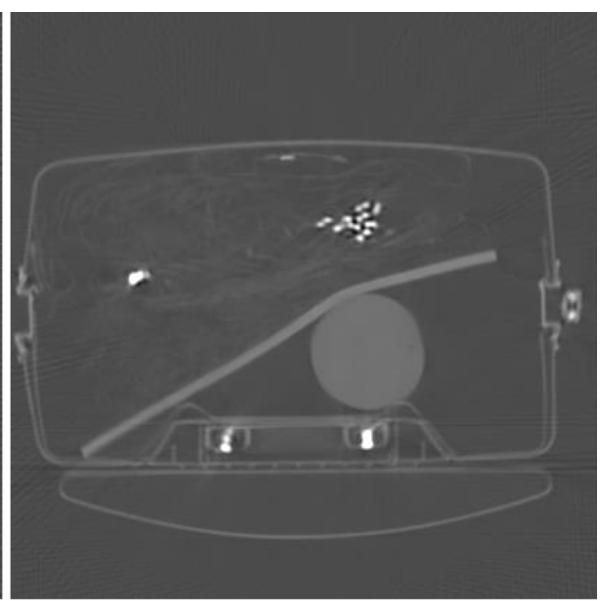

(b)

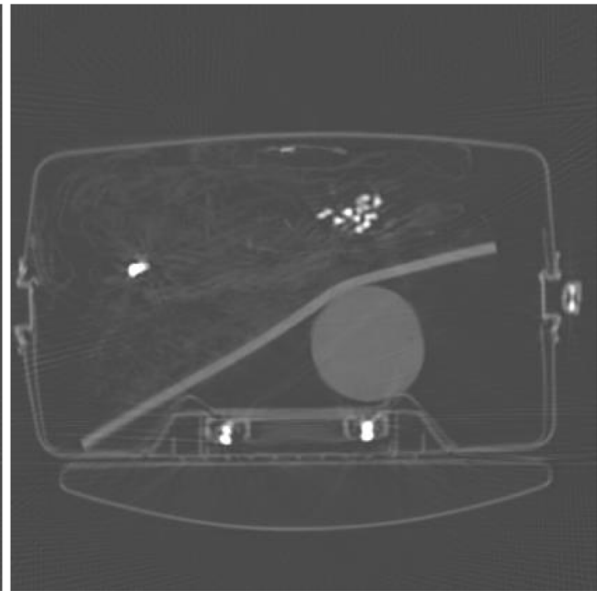

(d)

Fig. 1 Reconstruction of bag \#1. a Raw FBP; b TV-term only; c Non-negativity-term only; d Combined. Some artifacts are marked by the red arrows 


$$
\begin{gathered}
u_{i, j}=\frac{\partial T_{1}}{\partial y_{i, j}} \\
=\frac{\left(y_{i, j}-y_{i, j+1}\right)+\left(y_{i, j}-y_{i+1, j}\right)}{\sqrt{\left(y_{i, j}-y_{i, j+1}\right)^{2}+\left(y_{i, j}-y_{i+1, j}\right)^{2}}} \\
+\frac{y_{i, j}-y_{i, j-1}}{\sqrt{\left(y_{i, j-1}-y_{i, j}\right)^{2}+\left(y_{i, j-1}-y_{i+1, j-1}\right)^{2}}} \\
+\frac{y_{i, j}-y_{i-1, j}}{\sqrt{\left(y_{i-1, j}-y_{i-1, j+1}\right)^{2}+\left(y_{i-1, j}-y_{i, j}\right)^{2}}}
\end{gathered}
$$

When the quantity under a square root is zero, the gradient $u_{i, j}$ is not well-defined. In this case, we use the sub-gradient and assume the sub-gradient $u_{i, j}$ to be zero

$$
u_{i, j}=0
$$

The gradient of $T_{1}$ given in Formula (6) is with respect to the image pixels $y_{i, j}$. However, the gradient decent algorithm (Formula 4) requires the gradient of $T_{1}$ be with respect to the projections $P$.
Realizing that $Y$ is in the image domain, the algorithm variables are in the projection domain related by the mapping

$$
P=A Y
$$

where, $A$ represents the forward projection matrix. The matrix $A$ maps the image into its projections. This same matrix $A$ can map the gradient $U$ into the projection domain as $A U$, where the entries of $U$ are $u_{i, j}$. We have

$$
\nabla T_{1}(P)=A U
$$

\section{Minimization of the second term in the objective function (Formula 1)}

Let $Z=\min \{0, F(P)\}=\min \{0, X\}$ be the negative pixels in the FBP reconstruction $X$. The energy of the negative image pixels is the square of the $L_{2}$ norm of $Z$ given as

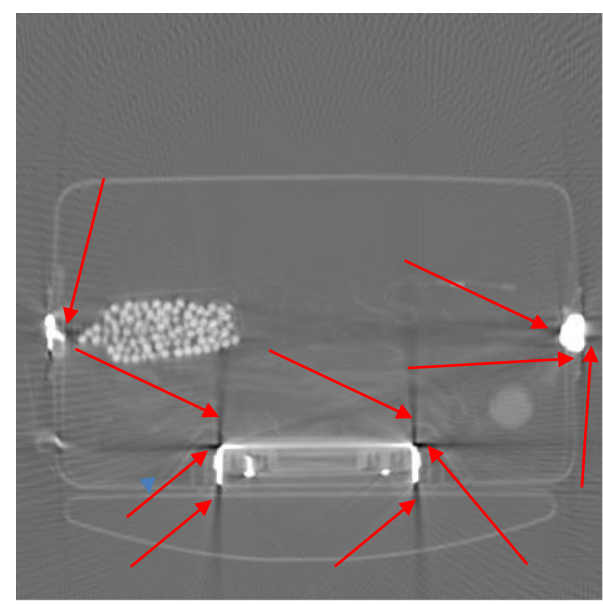

(a)

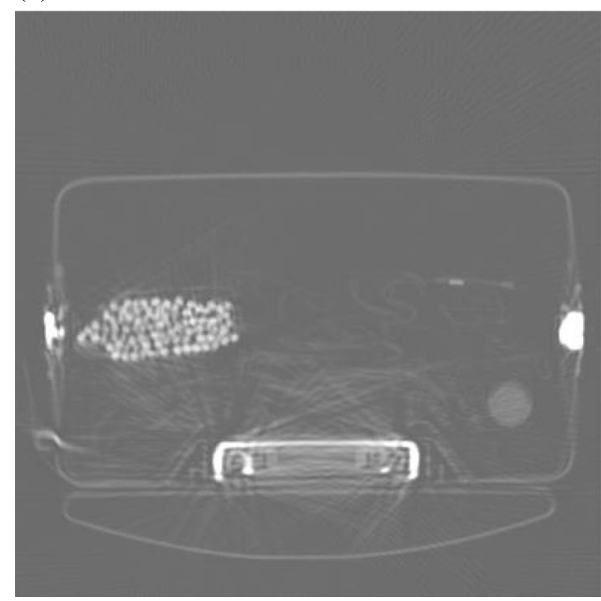

(c)

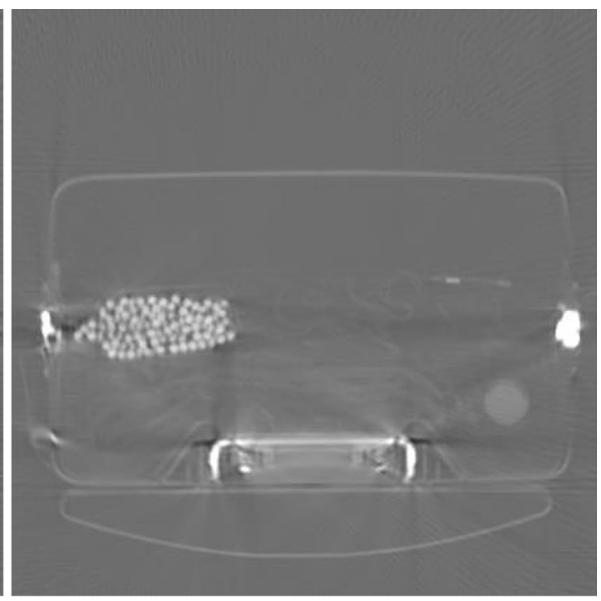

(b)

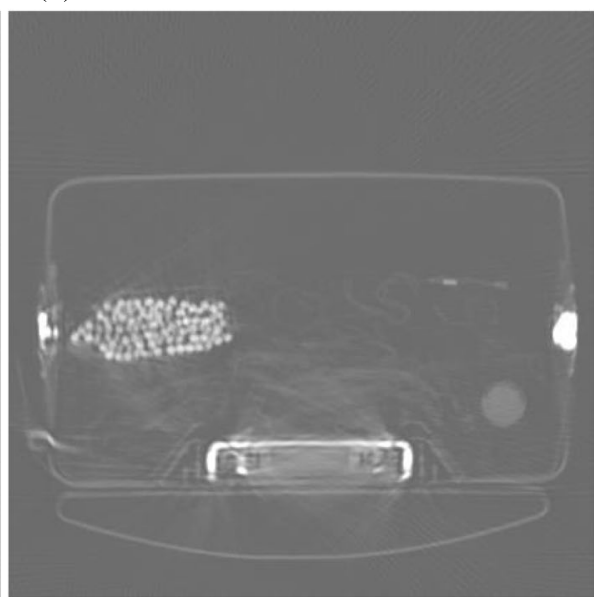

(d)

Fig. 2 Reconstruction of bag \#2. a Raw FBP; $\mathbf{b}$ TV-term only; c Non-negativity-term only; $\mathbf{d}$ Combined. Some artifacts are marked by the red arrows 


$$
T_{2}=\|Z\|_{2}^{2}=\|\min \{0, F P\}\|_{2}^{2}
$$

To find the gradient $\nabla T_{2}(P)$, that is, $\partial T_{2} / \partial p_{j}$, is not straightforward, because the 'min' function in Formula (10) makes $T_{2}$ non-differentiable. We can use the subdifferential concept to find the gradient of $\partial T_{2} / \partial p_{j}$ as [27]

$$
\nabla T_{2}=2 F^{T} \min \{0, F P\}=2 F^{T} Z
$$

where $F^{T}$ represents forward projection followed by ramp filtering. The operator $F^{T}$ maps an image into the projection domain.

\section{Proposed gradient descent algorithm to estimate $P_{M}$}

Combining the gradients above, the proposed gradient descent iterative algorithm (Formula 4) to estimate $P_{M}$ can given as

$$
P_{M}^{(k+1)}=P_{M}^{(k)}-D\left[\beta_{1} \tanh (A U)+\beta_{2} F^{T} Z\right]
$$

where $\tanh \left(\nabla T_{1}\right)$ is used in place of $\nabla T_{1}$ in Formula (4). The purpose of the hyperbolic tangent function 'tanh' is to hard limit the TV gradient values, making the iterative algorithm more stable.

The proposed algorithm is different from the commonly used iterative algorithms in the sense that the sinogram values in a common iterative reconstruction algorithm are never altered. Also, the proposed algorithm does not have a data fidelity term in the objective function (Formula 1). Our final image is obtained by the FBP algorithm, after $P_{M}$ is modified. The initial value of the metal affected part $P_{M}$ is the measured value.

We tested the proposed algorithm with some unknown airport bags, which contained some unknown metals. The projection sinograms were provided by the US Department of Homeland Security. The data sets were acquired with unknown $\mathrm{kVp}$ and unknown current

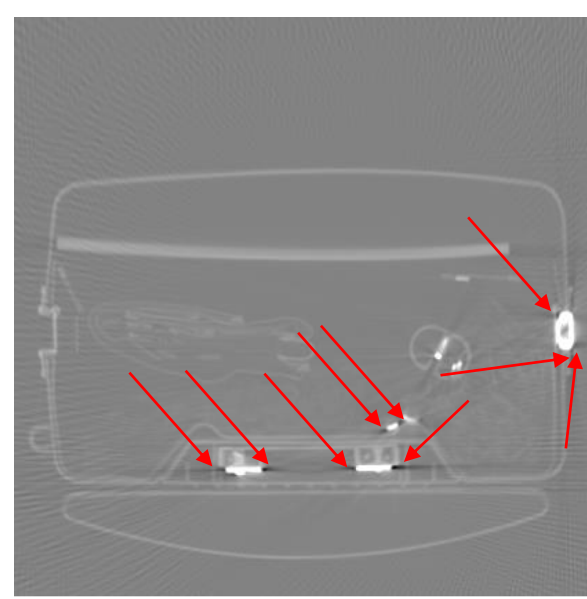

(a)

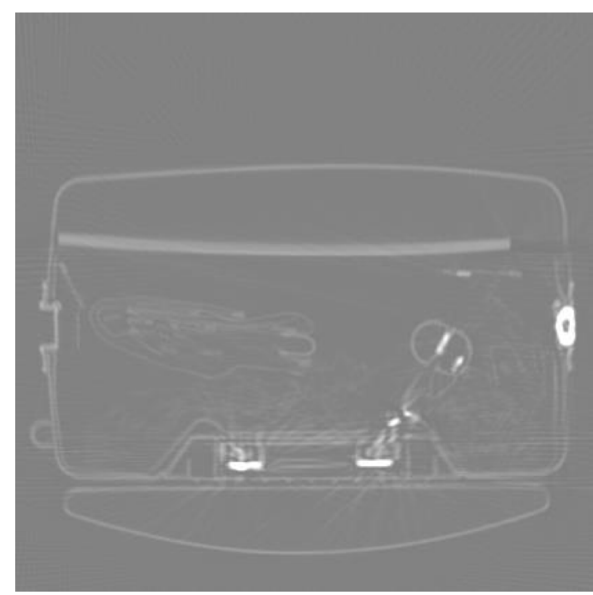

(c)

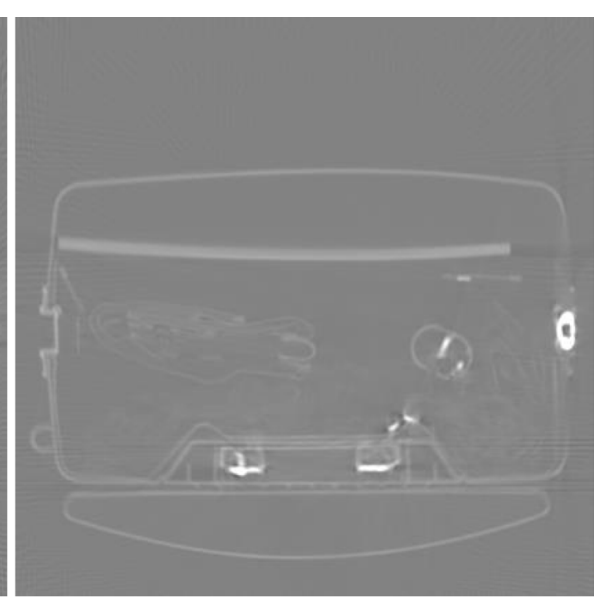

(b)

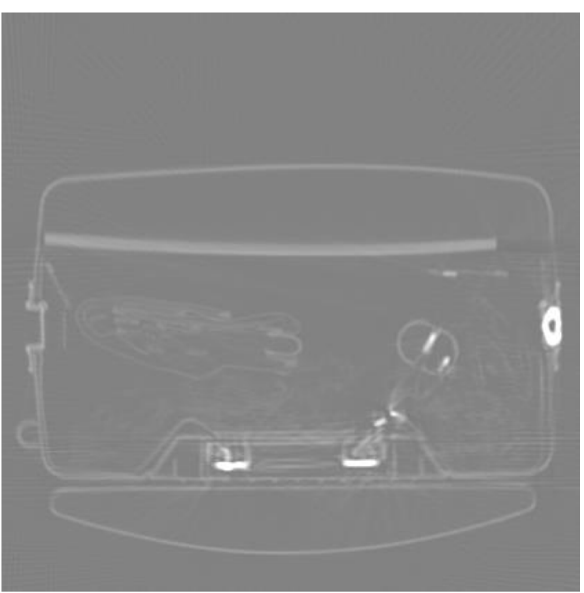

(d)

Fig. 3 Reconstruction of bag \#3. a Raw FBP; $\mathbf{b}$ TV-term only; c Non-negativity-term only; $\mathbf{d}$ Combined. Some artifacts are marked by the red arrows 
settings. The original sinograms were converted into parallel-beam geometry with 180 views over $180^{\circ}$ and with 597 detection channels (i.e., detector bins).

\section{Results}

Figures 1, 2, 3, 4 and 5 show the reconstruction results of 5 different unknown airport bags. Each figure consists of 4 images: the original raw FBP reconstruction; the TV-norm-only (with $\beta_{1}=0.004$ and $\beta_{2}=0$ ) reconstruction; the negativity-pixel-energy-only (with $\beta_{1}=0$ and $\beta_{2}=5$ ); the combined reconstruction (with $\beta_{1}=0.004$ and $\beta_{2}=5$ ). All images have the same display window. Black color represents negative values and white color represents large positive values. In order to visualize the metal artifacts, the display window is determined by the raw FBP reconstruction using the original measured sinogram. The display window is airport bag specific. The $1 / 3$ of the maximum raw FBP image value is mapped to the gray level 255 (white). Any value larger is set to 255. The metal pixels are much brighter than other pixels in the image. If we do not clip the very bright metal pixels, we cannot see other structures in the image. The minimum raw FBP value (which is negative) is mapped to the gray level 0 (black). The mapping is linear in the range below $1 / 3$ of the maximum value. All 4 images in the figure use the same gray-scale mapping. Therefore, back pixels are negative.

The number of iterations for all studies in this paper was 400 in the gradient descent algorithm. The image array size was $420 \times 420$, and the pixel size was $0.92 \mathrm{~mm}$. The proposed algorithm was implemented using MATLAB $^{\circ}$, and the computational time for 400 iterations was $145 \mathrm{~s}$.

Because the ground truth is unknown to us, it is inappropriate to perform quantitative evaluations. In this paper, we only make human visual inspections on the reconstructions, focusing on the severeness of the artifacts. Two evaluation metrics were used. One metric

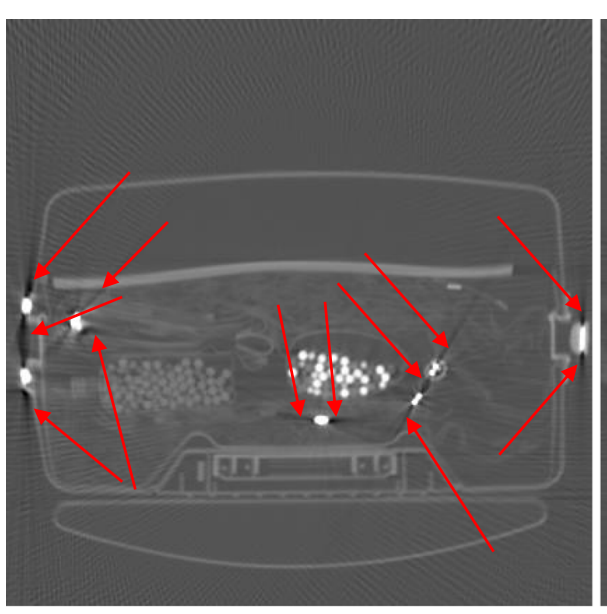

(a)

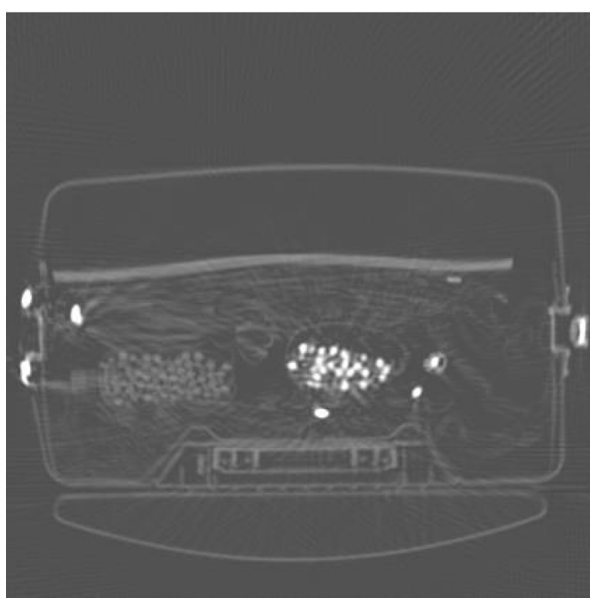

(c)

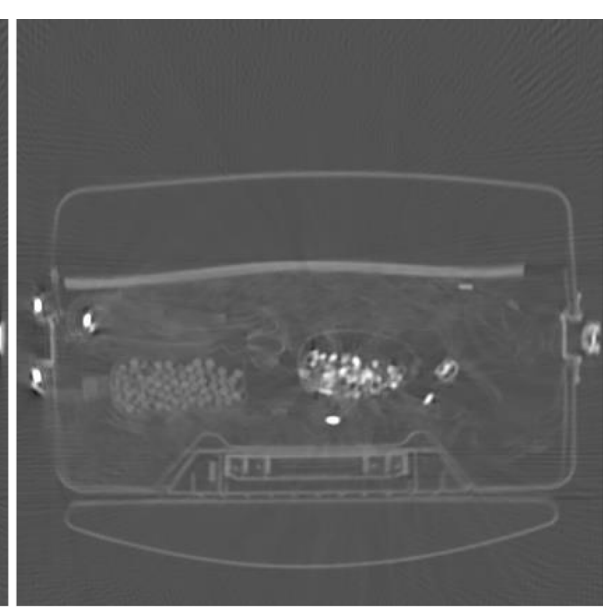

(b)

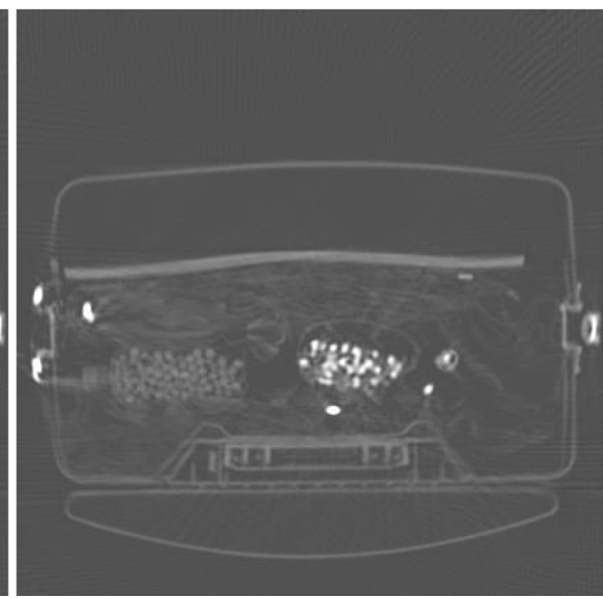

(d)

Fig. 4 Reconstruction of bag \#4. a Raw FBP; $\mathbf{b}$ TV-term only; c Non-negativity-term only; $\mathbf{d}$ Combined. Some artifacts are marked by the red arrows 


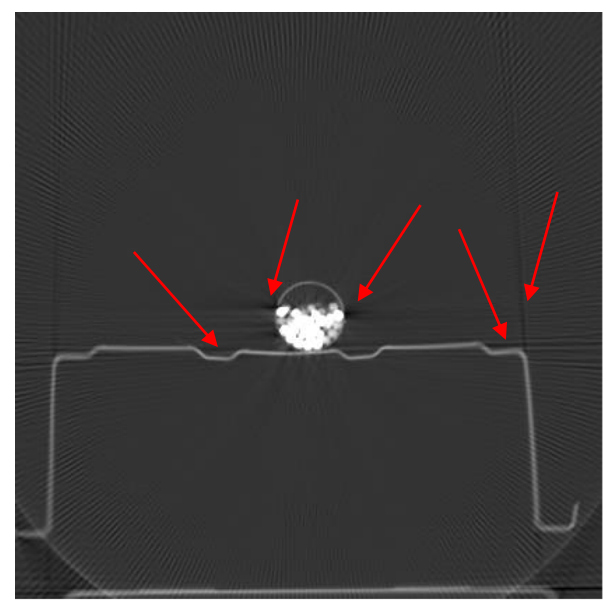

(a)

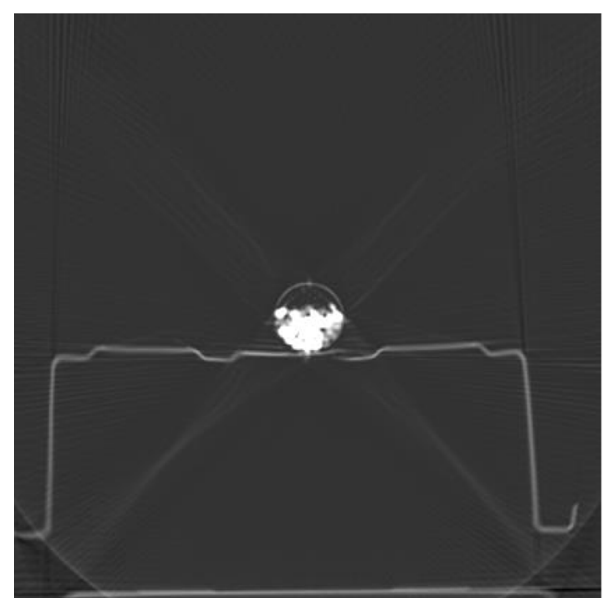

(c)

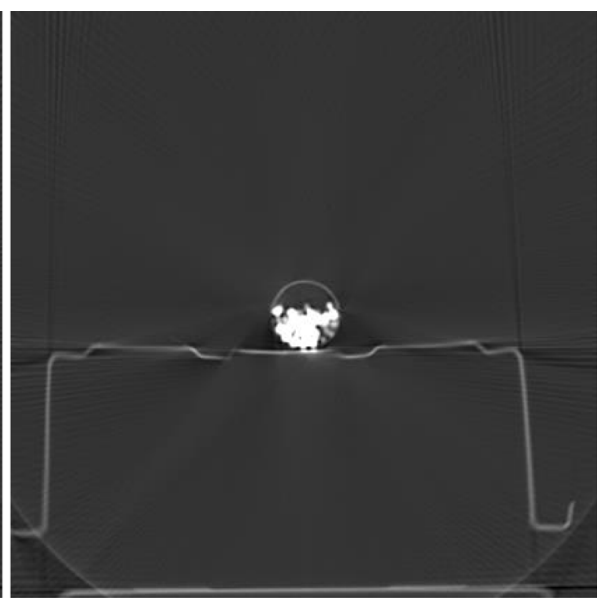

(b)

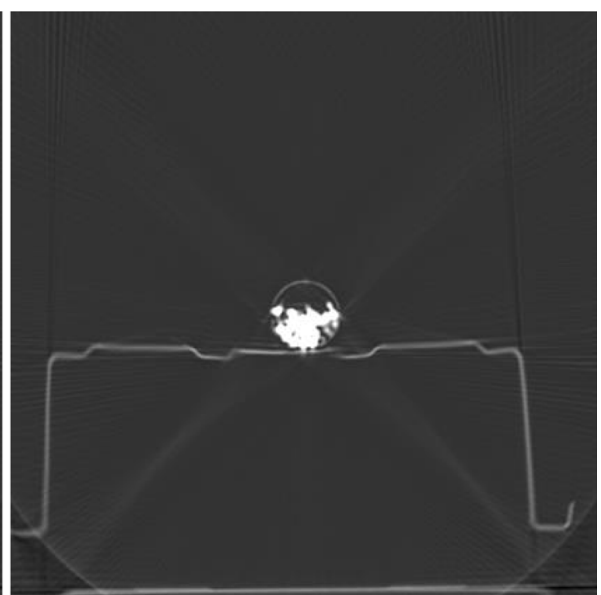

(d)

Fig. 5 Reconstruction of bag \#5. a Raw FBP; b TV-term only; c Non-negativity-term only; d Combined. Some artifacts are marked by the red arrows

was the TV norm of the reconstructed image with metals removed. The other metric was the energy of the negative pixels in the reconstructed image.

It is seen that all raw FBP images (shown at the upperleft conner) contain severe black streaking artifacts, indicating streaks of negative values. When the objective function only has the TV-norm term, that is, $\beta_{2}=0$, most black streaks are removed from the images (shown at the upper-right conner); unfortunately, many lowcontrast structures are lost at the same time. The lowerleft images are obtained using only the negative-pixelenergy minimization (i.e., $\beta_{1}=0$ ), and the black streaking artifacts are effectively removed from the resulting reconstructions. Compared with the upper-right images, the lower-left images look noisier and may contain some new bright streaking artifacts. If both of the two terms are used in the objective function (Formula 1), the resultant images are shown at the lower-right conner, which enjoy the benefits from both terms in the objective function (Formula 1).

In Fig. 5, the metal reduction methods fail to remove the dark streaking artifacts. On the other hand, Fig. 6 shows better results for the same airport bag. The different results in these two figures are mainly due to the different setup in metal image segmentation. In Figs. 1, 2, 3,4 and 5 , the FBP image pixels are segmented into the metal image if the image values are greater than $t=1 / 3$ of the maximum image value. However, in Fig. 6, the FBP image pixels are segmented into the metal image if the image values are greater than $1 / 10$ of the maximum image value. The change of the segmentation threshold results in different metal maps, as illustrated in Fig. 7. After changing the threshold value $t$ from $1 / 3$ to $1 / 10$, we must change $\beta_{1}=0.004$ to a much smaller value $\beta_{1}=$ 0.002 , otherwise the resultant image is too smooth and lots of image details disappear. 


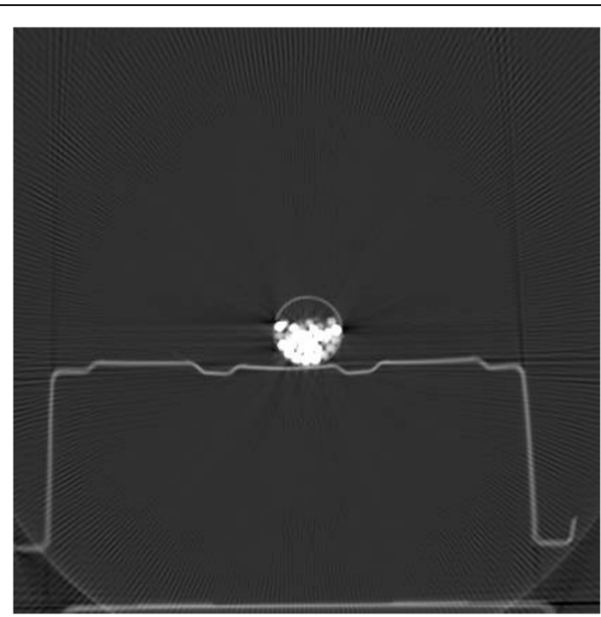

(a)

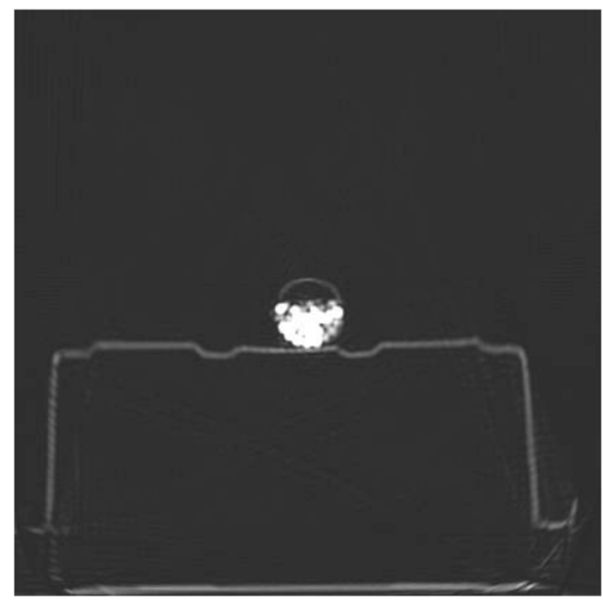

(c)

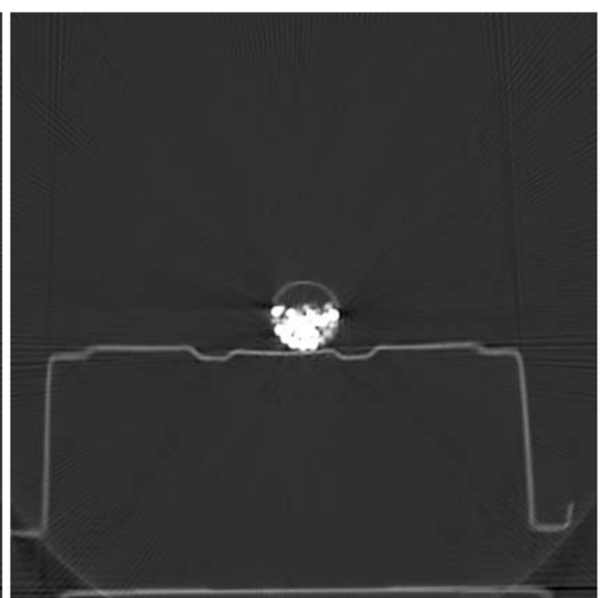

(b)

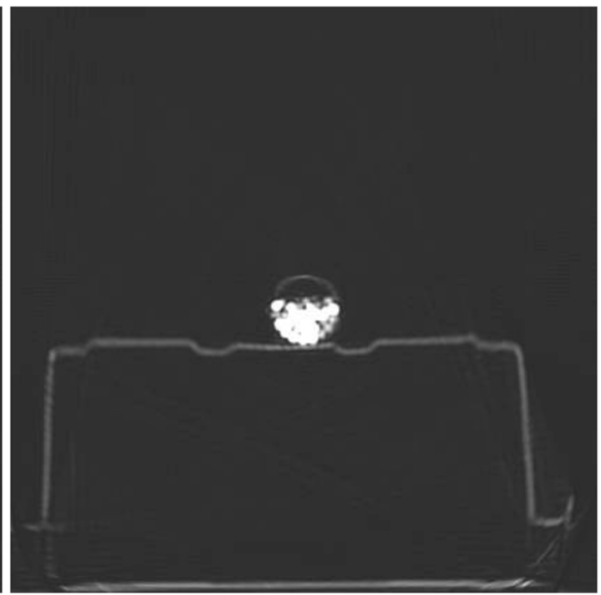

(d)

Fig. 6 Reconstruction of bag \#5 (New threshold value to segment the metal image and new $\beta_{1}=0.0002$ ). a Raw FBP; b TV-term only; c Nonnegativity-term only; $\mathbf{d}$ Combined

Tables 1, 2, 3, 4, 5 and 6 list the maximum and minimum values for each image in Figs. 1, 2, 3, 4, 5 and 6, respectively. It is noticed that the images generated from the processed sinograms still have negative image pixels. The residual negative pixels are most likely due to the angular aliasing artifacts because we use only 180 views in our sinogram data. A typical X-ray CT scan has approximately 1000 views. The tables also list the evaluation metrics: the TV norm of the reconstructed image with metals removed and the energy of the negative pixels in the reconstructed image.

Figure 8 compares bag \#1 sinograms before and after processing. Figure $8(\mathrm{a})$ is for the case of $\beta_{1}=0.004$ and $\beta_{2}=5$. Figure $8(\mathrm{~b})$ represents the raw measurements. The difference image $[(a)-(b)]$ is shown at Fig. 8(c). It is observed that most sinogram values are not altered by the iterative algorithm.

\section{Discussion}

This paper combines two methods into one. These two methods use two different objective functions. They try to reduce the metal artifacts by using two different strategies. One method tries to reduce the artifacts that have oscillations; the other method tries to reduce the artifacts that have negative overshoots. By combining these two methods, we have a better chance to reduce metal artifacts that have both oscillating and negative overshoots features. One potential limitation is that those two methods may work in the opposite ways, making none of the methods effective. Another potential limitation is that the combined method has some hyper 


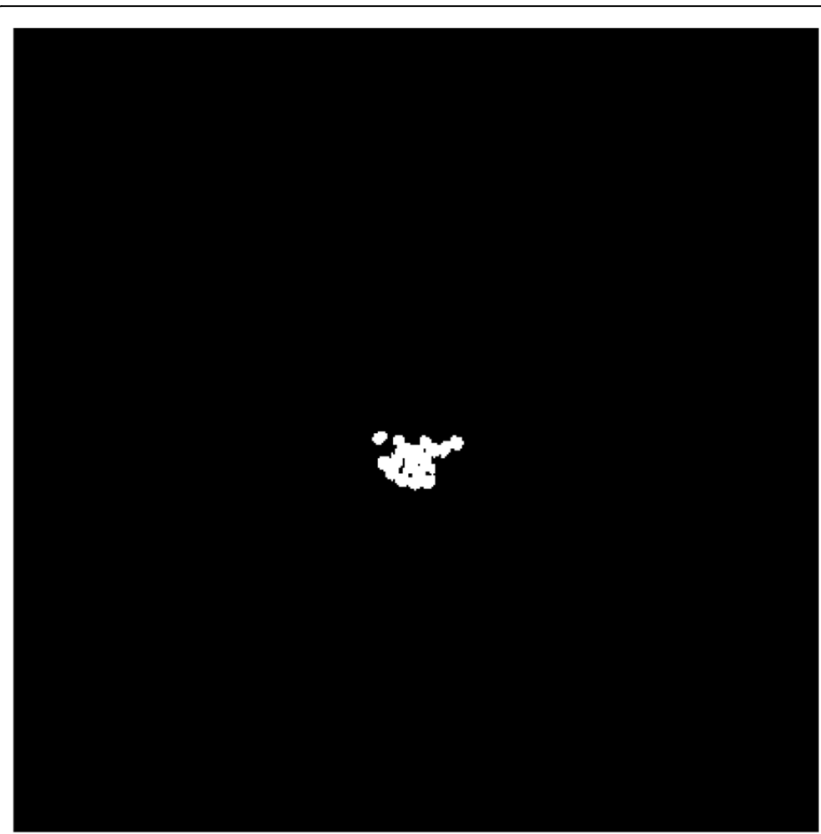

(a)

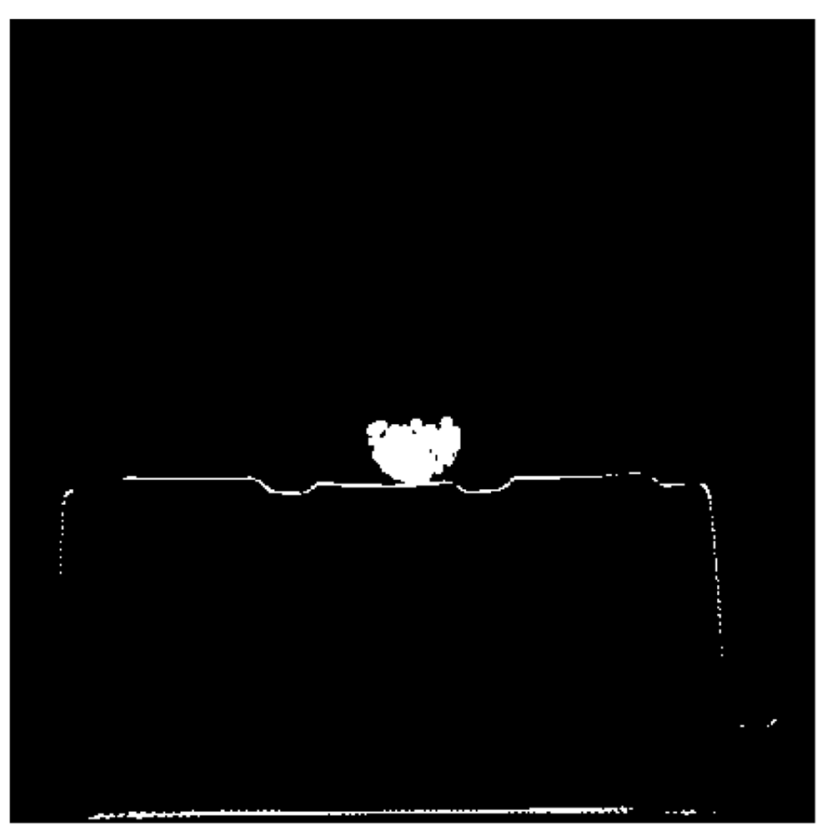

(b)

Fig. 7 Metal images for bag \#5. a The segmentation threshold $t=1 / 3$ of the maximum raw FBP image pixel value; $\mathbf{b}$ The segmentation threshold $t=1 / 10$ of the maximum raw FBP image pixel value

Table 1 Values of maximum, minimum, metal-free region TV, and NPE in reconstructions of bag \#1

\begin{tabular}{lllllll}
\hline Iterations & $\boldsymbol{\beta}_{\mathbf{1}}$ & $\boldsymbol{\beta}_{\mathbf{2}}$ & Min & Max & TV & NPE \\
\hline 0, raw FBP & NA & NA & -0.1591 & 1.2781 & 1501.4 & 3.1231 \\
1000 & 0.004 & 0 & -0.1365 & 1.0643 & 835.6338 & 0.3426 \\
1000 & 0 & 5 & -0.0133 & 1.1837 & 1014.6 & 0.0631 \\
1000 & 0.004 & 5 & -0.0134 & 1.1408 & 869.5945 & 0.0712 \\
\hline
\end{tabular}

Table 2 Values of maximum, minimum, metal-free region TV, and NPE in reconstructions of bag \#2

\begin{tabular}{lllllll}
\hline Iterations & $\boldsymbol{\beta}_{\mathbf{1}}$ & $\boldsymbol{\beta}_{\mathbf{2}}$ & Min & Max & TV & NPE \\
\hline 0, raw FBP & NA & NA & -0.3000 & 1.3420 & 1148.4 & 8.3468 \\
1000 & 0.004 & 0 & -0.11825 & 1.1570 & 834.2642 & 3.1681 \\
1000 & 0 & 5 & -0.0196 & 1.3585 & 1150.3 & 0.1340 \\
1000 & 0.004 & 5 & -0.0206 & 1.3130 & 906.2427 & 0.1476 \\
\hline
\end{tabular}

Table 3 Values of maximum, minimum, metal-free region TV, and NPE in reconstructions of bag \#3

\begin{tabular}{lllllll}
\hline Iterations & $\boldsymbol{\beta}_{\boldsymbol{1}}$ & $\boldsymbol{\beta}_{\boldsymbol{2}}$ & Min & Max & TV & NPE \\
\hline 0, raw FBP & NA & NA & -0.3776 & 1.2154 & 1144.3 & 4.7415 \\
1000 & 0.004 & 0 & -0.0843 & 1.0329 & 891.2476 & 1.6540 \\
1000 & 0 & 5 & -0.0191 & 1.1270 & 1025.8 & 0.3326 \\
1000 & 0.004 & 5 & -0.0223 & 1.0872 & 964.3871 & 0.3294 \\
\hline
\end{tabular}

Table 4 Values of maximum, minimum, metal-free region TV, and NPE in reconstructions of bag \#4

\begin{tabular}{lllllll}
\hline Iterations & $\boldsymbol{\beta}_{\mathbf{1}}$ & $\boldsymbol{\beta}_{\mathbf{2}}$ & Min & Max & TV & NPE \\
\hline 0, raw FBP & NA & NA & -0.1868 & 1.3204 & 1379.1 & 1.9616 \\
1000 & 0.004 & 0 & -0.0757 & 1.2775 & 1006.9 & 0.4434 \\
1000 & 0 & 5 & -0.0155 & 1.3992 & 1226.9 & 0.1255 \\
1000 & 0.004 & 5 & -0.0164 & 1.4060 & 1062.4 & 0.1379 \\
\hline
\end{tabular}

Table 5 Values of maximum, minimum, metal-free region TV, and NPE in reconstructions of bag \#5

\begin{tabular}{lllllll}
\hline Iterations & $\boldsymbol{\beta}_{\mathbf{1}}$ & $\boldsymbol{\beta}_{\mathbf{2}}$ & Min & Max & TV & NPE \\
\hline 0, raw FBP & NA & NA & -0.0347 & 0.4676 & 472.6624 & 0.3366 \\
1000 & 0.004 & 0 & -0.0371 & 0.5137 & 220.4515 & 0.0558 \\
1000 & 0 & 5 & -0.0340 & 0.5487 & 225.6903 & 0.0201 \\
1000 & 0.004 & 5 & -0.0344 & 0.5041 & 220.1787 & 0.0222 \\
\hline
\end{tabular}

Table 6 Values of maximum, minimum, metal-free region TV, and NPE in reconstructions of bag \#5 (New threshold value to segment the metal image and new $\beta_{1}=0.0002$ )

\begin{tabular}{lllllll}
\hline Iterations & $\boldsymbol{\beta}_{\mathbf{1}}$ & $\boldsymbol{\beta}_{\mathbf{2}}$ & Min & Max & TV & NPE \\
\hline 0, raw FBP & NA & NA & -0.0347 & 0.4676 & 472.6624 & 0.3366 \\
1000 & 0.0002 & 0 & -0.0342 & 0.5251 & 165.2475 & 0.0190 \\
1000 & 0 & 5 & -0.0030 & 0.5243 & 180.0589 & 0.00015141 \\
1000 & 0.0002 & 5 & -0.0031 & 0.5252 & 144.7916 & 0.0011 \\
\hline
\end{tabular}




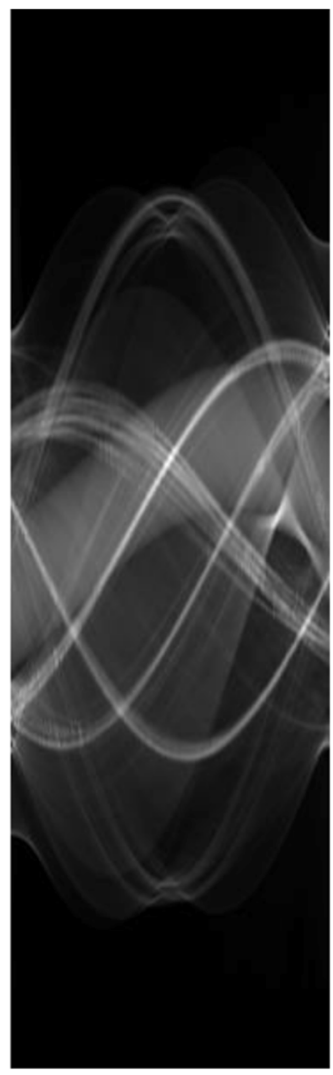

(a)

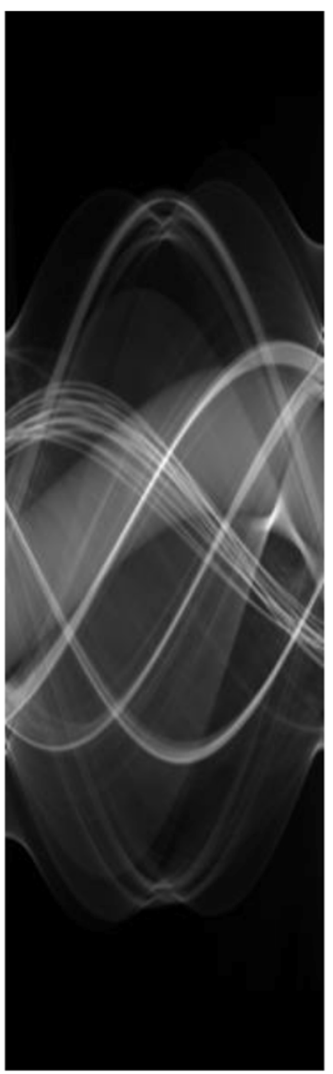

(b)

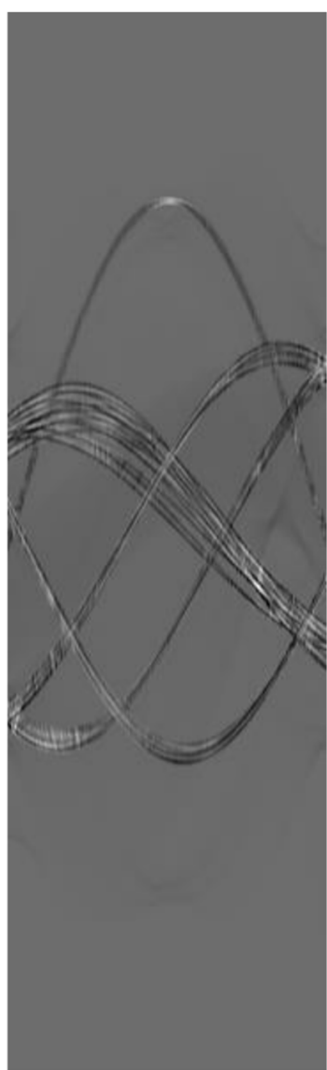

(c)

Fig. 8 Sinograms of bag \#1. a Processed with $\beta_{1}=0.004$ and $\beta_{2}=5$; b Raw; c Difference

parameters $\beta 1$ and $\beta 2$ to be adjusted. There are no general rules to set them.

The ratio of these two parameters determines which objective function should be more dominating. Their values also affect the convergence rate of the gradient descent algorithm. Usually, larger values of $\beta_{1}$ and $\beta_{2}$ result in fast convergence. If their values are too large, the gradient descent algorithm may diverge. There is no clear relationship between the beta values and the threshold value. The values of $\beta_{1}$ and $\beta_{2}$ should be set for every new data set and every new object. The value of $\beta_{2}$ may not be sensitive to the data set or object. There are no general rules how to set these two hyper parameters. It is observed from Tables 1, 2, 3, 4, 5 to 6 that the TV norm values $T_{1}$ are much larger than the energy of negative pixel energy (NPE) values $T_{2}$. Therefor, $\beta_{1}$ is much smaller than $\beta_{2}$.

The setting of the hyper parameters is data dependent. One setting works well for one object but does not work well for another object. This newer value of $\beta_{1}$ for bag \#5 did not work well for other data sets. The hyper parameters are set by trial-anderror for each case.
From our experimental results, there is no significant difference between the results of non-negativity-term only and combined. The minimum and maximum values of the two results are also very close. This observation implies that among the two methods, the method to reduce the negative overshoot is more effective than the TV minimization method for the cases presented.

\section{Conclusions}

This paper proposes a projection-domain iterative algorithm to estimate metal affected projections using an image-domain objective function. The objective function does not have a data fidelity term. It consists of the TV norm and the energy of the negative pixels. Real CT scans with unknown objects and unknown metals are used to test the feasibility of the proposed algorithm. The metals cause severe streaking artifacts in the raw FBP reconstructions. Those streaking artifacts are successfully removed by the proposed algorithm.

Results of two special cases, $\beta_{1}=0$ and $\beta_{2}=0$, are also compared. When $\beta_{2}=0$, the algorithm only minimizes the image TV norm. The images with TV norm minimization tend to be oversmoothed, and some low 
contrast structures may get lost. When $\beta_{1}=0$, the algorithm only minimizes the image NPE. The images with NPE minimization contain more low contrast structures but may be noisy. When both terms are used in the objective function (1), the final images are more balanced between details and noise control. In all three cases discussed above, the metal streaking artifacts are effectively reduced.

\section{Abbreviations}

CT: Computed tomography; FBP: Filtered backprojection; NPE: Negative pixel energy; TV: Total variation

\section{Acknowledgements}

The Reconstruction Initiative Dataset used in the research related to this publication was generated and provided by Awareness and Localization of Explosives-Related Threats, a Department of Homeland Security Center of Excellence. The views and conclusions contained in this document are those of the authors and should not be interpreted as necessarily representing the official policies, either expressed or implied, of the U.S. Department of Homeland Security. In this paper, we downgrade the spatial resolution of the original data on purpose.

\section{Author's contributions}

GLZ is the only author. The author read and approved the final manuscript.

\section{Funding}

This research is partially supported by NIH, No. R15EB024283.

\section{Availability of data and materials}

Not applicable.

\section{Declaration}

\section{Competing interests}

The author has any competing interests in the manuscript.

Received: 29 June 2021 Accepted: 14 October 2021

Published online: 02 January 2022

\section{References}

1. Curry TI, Dowdey JE, Murray RC Jr (1990) Christensen's physics of diagnostic radiology, 4th edn. Lea \& Febiger, Philadelphia, pp 61-69

2. Boas FE, Fleischmann D (2011) Evaluation of two iterative techniques for reducing metal artifacts in computed tomography. Radiology 259(3):894902. https://doi.org/10.1148/radiol.11101782

3. Barrett JF, Keat N (2004) Artifacts in CT: recognition and avoidance. RadioGraphics 24(6):1679-1691. https://doi.org/10.1148/rg.246045065

4. Matsumoto K, Jinzaki M, Tanami Y, Ueno A, Yamada M, Kuribayashi S (2011) Virtual monochromatic spectral imaging with fast kilovoltage switching: improved image quality as compared with that obtained with conventional 120-kVp CT. Radiology 259(1):257-262. https://doi.org/10.1148/radiol.111 00978

5. Medoff BP, Brody WR, Nassi M, Macovski A (1983) Iterative convolution backprojection algorithms for image reconstruction from limited data. J Opt Soc Amer 73(11):1493-1500. https://doi.org/10.1364/JOSA.73.001493

6. Wang G, Snyder DL, O'Sullivan JA, Vannier MW (1996) Iterative deblurring for CT metal artifact reduction. IEEE Trans Med Imag 15(5):657-664. https:// doi.org/10.1109/42.538943

7. Wang G, Vannier MW, Cheng CP (1999) Iterative X-ray cone-beam tomography for metal artifact reduction and local region reconstruction. Microsc Microanal 5(1):58-65. https://doi.org/10.1017/S1431927699000057

8. Wang G, Frei T, Vannier MW (2000) Fast iterative algorithm for metal artifact reduction in X-ray CT. Acad Radiol 7(8):607-614. https://doi.org/10.1016/S1 076-6332(00)80576-0

9. August J, Kanade T (2004) Fast streaking artifact reduction in CT using constrained optimization in metal masks. In: Barillot C, Haynor DR, Hellier P (eds) Medical image computing and computer-assisted intervention MICCAI 2004, 7th international conference, Saint-Malo, France, September
26-29, 2004. Springer, Heidelberg, pp 1044-1045. https://doi.org/10.1007/ 978-3-540-30136-3_130

10. De Man B (2008) Method and apparatus for the reduction of artifacts in computed tomography images. US patent 7444010, 28 Oct 2008

11. Luzhbin D, Wu J (2020) Model image-based metal artifact reduction for computed tomography. J Digit Imag 33(1):71-82. https://doi.org/10.1007/s1 0278-019-00210-6

12. Kubo Y, Ito K, Sone M, Nagasawa H, Onishi Y, Umakoshi N, et al (2020) Diagnostic value of model-based iterative reconstruction combined with a metal artifact reduction algorithm during $C T$ of the oral cavity. Amer J Neuroradiol 41(11):2132-2138. https://doi.org/10.3174/ajnr.A6767

13. Liu JJ, Watt-Smith SR, Smith SM (2005) Metal artifact reduction for CT based on sinusoidal description. J X-Ray Sci Technol 13(2):85-96

14. Gu JW, Zhang L, Yu GQ, Xing YX, Chen ZQ (2006) X-ray CT metal artifacts reduction through curvature based sinogram inpainting. J X-Ray Sci Technol 14(2):73-82

15. Zhang YB, Zhang LF, Zhu XR, Lee AK, Chambers M, Dong L (2007) Reducing metal artifacts in cone-beam CT images by preprocessing projection data. Int J Radiat Oncol Biol Phys 67(3):924-932. https://doi.org/10.1016/j.jprobp.2 006.09.045

16. Joemai RMS, De Bruin PW, Veldkamp WJH, Geleijns J (2012) Metal artifact reduction for $\mathrm{CT}$ : development, implementation, and clinical comparison of a generic and a scanner-specific technique. Med Phys 39(2):1125-1132. https://doi.org/10.1118/1.3679863

17. Kratz B, Weyers I, Buzug TM (2012) A fully 3D approach for metal artifact reduction in computed tomography. Med Phys 39(11):7042-7054. https:// doi.org/10.1118/1.4762289

18. Yu LF, Li H, Mueller J, Kofler J, Liu X, Primak AN et al (2009) Metal artifact reduction from reformatted projections for hip prostheses in multislice helical computed tomography: techniques and initial clinical results. Investig Radiol 44(11):691-696. https://doi.org/10.1097/RLI.0b013e3181b0a2f9

19. Yu HY, Zeng K, Bharkhada DK, Wang G, Madsen MT, Saba O, et al (2007) A segmentation-based method for metal artifact reduction. Acad Radiol 14(4): 495-504. https://doi.org/10.1016/j.acra.2006.12.015

20. Karimi S, Cosman P, Wald C, Martz H (2012) Segmentation of artifacts and anatomy in CT metal artifact reduction. Med Phys 39(10):5857-5868. https:// doi.org/10.1118/1.4749931

21. Xue H, Zhang L, Xiao YS, Chen ZQ, Xing YX (2009) Metal artifact reduction in dual energy $C T$ by sinogram segmentation based on active contour model and TV inpainting. In: Abstracts of the 2009 IEEE nuclear science symposium conference record, IEEE, Orlando, 24 Oct-1 Nov 2009

22. Zhang Y, Pu YF, Hu JR, Liu Y, Zhou JL (2011) A new CT metal artifacts reduction algorithm based on fractional-order sinogram inpainting. $J X$-Ray Sci Technol 19(3):373-384. https://doi.org/10.3233/XST-2011-0300

23. Zhang XM, Lei X (2013) Sequentially reweighted TV minimization for CT metal artifact reduction. Med Phys 40(7):071907. https://doi.org/10.1118/1.4 811129

24. Manhart M, Psychogios M, Amelung N, Knauth M, Rohkohl C (2017) Improved metal artifact reduction via image quality metric optimization. In: abstracts of the 14th international meeting on fully 3D image reconstruction in radiology and nuclear medicine, Xi'an, China, 19-23 June 2017

25. Zeng GL (2020) Projection-domain iteration to estimate unreliable measurements. Vis Comput Ind Biomed Art 3(1):16. https://doi.org/10.1186/ s42492-020-00054-w

26. Zeng GL, Zeng M (2021) Reducing metal artifacts by restricting negative pixels. Vis Comput Ind Biomed Art 4(1):17. https://doi.org/10.1186/s42492021-00083-z

27. Calafiore GC, El Ghaoui L (2014) Optimization models. Cambridge University Press, Cambridge. https://doi.org/10.1017/CBO9781107279667

\section{Publisher's Note}

Springer Nature remains neutral with regard to jurisdictional claims in published maps and institutional affiliations. 